\section{Induction de la tolérance centrale dans le thymus par le facteur de transcription Aire}

Noëlla Lopes, Pierre Ferrier, Magali Irla

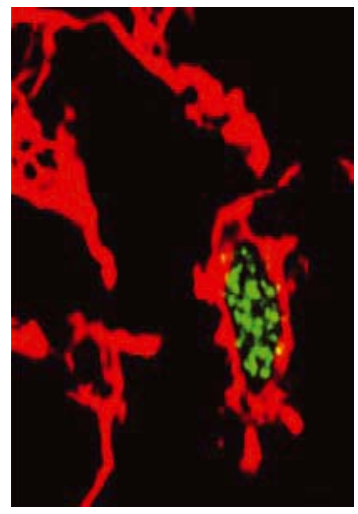

Centre d'Immunologie de Marseille-Luminy, Aix Marseille Université UM2, Inserm, U1104, CNRS UMR7280, 13288 Marseille, France. lopes@ciml.univ-mrs.fr ferrier@ciml.univ-mrs.fr magali.irla@inserm.fr

Nous discutons également d'autres rôles, récemment décrits, de ce facteur de transcription dans l'induction de la tolérance centrale. <

\section{Différenciation des cellules épithéliales médullaires thymiques}

Les cellules épithéliales médullaires thymiques (mTEC) jouent un rôle critique dans l'induction de la tolérance centrale des cellules $T$, grâce à leur capacité unique d'exprimer des milliers d'antigènes spécifiques de tissus périphériques (TRA, tissue-restricted antigen) [1]. Cette expression conduit à l'élimination de cellules T autoréactives potentiellement dangereuses pour l'intégrité de nos tissus et permet la génération de cellules T régulatrices naturelles (nTreg). Ces dernières ont la capacité de contrôler en périphérie l'activité de cellules T autoréactives qui ont échappé aux processus de sélection thymique [2]. L'expression de nombreux TRA est régulée par le facteur de transcription Aire (autoimmune regulator) [3]. Chez l'embryon, de récents travaux ont établi que les mT\&C Aire ${ }^{+}$dérivent de cellules progénitrices qui expriment fortement les protéines des jonctions serrées, claudines 3 et 4 , ainsi que le marqueur de cellules souches embryonnaires SSEA-1 (stage-specific embryonic antigen 1) (Figure 1) [4, 5]. Chez l'adulte, ces progéniteurs persistent mais perdent progressivement cette capacité d'autorenouvellement. De manière intéressante, l'implantation de ces cellules dans un environnement thymique dépourvu de mTEC restaure la formation d'un compartiment médullaire fonctionnel capable d'induire la tolérance des cellules T [5]. Ces résultats ouvrent de nouvelles perspectives thérapeutiques visant à améliorer la fonction thymique. Les travaux des quinze dernières années ont établi que les mTEC constituent une population cellulaire hétérogène. Ces cellules subissent en effet plusieurs étapes de différenciation caractérisées par la surexpression à la surface cellulaire des molécules du complexe majeur d'histocompatibilité de classe II (CMH II) et de la molécule de costimulation CD80 (Figure 1) [6]. Seules $25 \%$ des mTEC expriment Aire et sont dites «matures » $[7,8]$. Nous avons montré que leur différenciation et leur homéostasie sont contrôlées par des interactions spécifiques de l'antigène avec les thymocytes $\operatorname{CD} 4^{+}$dans le thymus postnatal [9-11]. Les mTEC matures expriment une grande diversité de TRA, ne prolifèrent pas et ont une durée de vie courte [7, 12]. Cette sous-population cellulaire était considérée jusqu'à présent comme correspondant au stade final de maturation des mTદC. Cependant, des études de traçabilité du destin cellulaire (fate mapping) ${ }^{1}$, qui ont permis de suivre au cours du temps les cellules ayant exprimé Aire antérieurement, ont montré l'existence d'une population de mTEC dite «post-Aire » (Figure 1) [13, 14]. Ces cellules se caractérisent par une expression modérée des molécules de CMH II et CD80 ainsi que par la perte totale d'expression de Aire. Elles présentent aussi la particularité d'exprimer plusieurs antigènes spécifiques des kératinocytes [15]. La perte de Aire s'accompagne d'une

${ }^{1}$ La technique du «fate mapping » permet de tracer le destin des cellules au cours du développement, grâce à l'expression d'un gène rapporteur, celle-ci étant transmise à la descendance. 


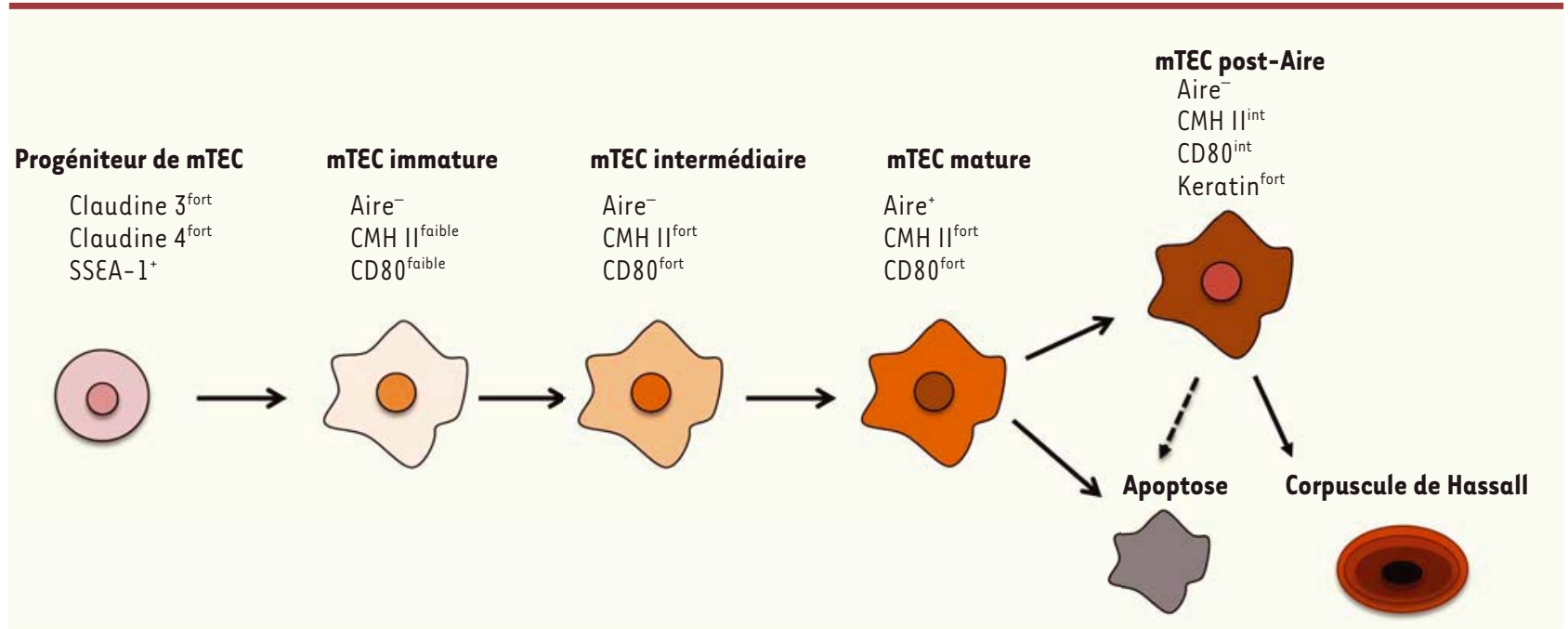

Figure 1. Modèle de différenciation des mTEC. Les cellules progénitrices des mTEC expriment les protéines des jonctions serrées, claudines 3 et 4 ainsi que le marqueur de cellules souches embryonnaires SSEA-1. Les différents stades de maturation des mTEC se caractérisent par une expression différentielle des molécules de CMH II, de la molécule de costimulation CD80 et du facteur de transcription Aire. La moitié des mTEC matures meurent par apoptose et l'autre moitié se différencient en $\mathrm{mTEC}$ post-Aire. Ces dernières se caractérisent par la perte d'expression de Aire, une diminution d'expression des molécules de CMH II, CD80 et par une forte expression des antigènes spécifiques des kératinocytes. Enfin, ces cellules au stade terminal de différenciation s'organisent en corpuscules de Hassall. Certaines d'entre elles pourraient être cependant susceptibles de mourir par apoptose (flèche en pointillé).

expression réduite de TRA, pas d'une abolition totale [14]. II est donc plausible que les $\mathrm{mTEC}$ post-Aire continuent à contribuer à l'induction de la tolérance centrale bien que ce rôle reste à établir. Enfin, les mTદC post-Aire perdent leurs noyaux et fusionnent pour former des corpuscules de Hassall. Ces structures sont identifiées à l'aide des marqueurs spécifiques de cellules différenciées de l'épiderme tels que les cytokératines 6 et 10, l'involucrine, le marqueur LEKTI (lympho-epithelial Kazal type related inhibitor), ainsi que les desmogléines 1 et 3 (Figure 1) $[15,16]$. Chez l'homme, les corpuscules de Hassall ont le potentiel d'induire le développement de cellules nTreg [17]. Leur(s) rôle(s) chez la souris reste(nt) à l'heure actuelle inconnu(s). II reste à déterminer si les mTEC post-Aire dans leur totalité deviennent des corpuscules de Hassall ou si une fraction d'entre elles meurent par apoptose (Figure 1). Il est intéressant de noter que la différenciation terminale des mTEC est contrôlée par le facteur Aire [18, 19]. En effet, son absence entraîne des perturbations du compartiment médullaire qui se caractérisent par une réduction du nombre de corpuscules de Hassall et une accumulation de mTEC matures présentant des altérations morphologiques [7, 16]. Ainsi, Aire influence le processus dynamique de la différenciation des mTEC. Ces études révèlent donc que l'activité de Aire s'étend au-delà du rôle bien établi de régulateur de l'expression des TRA dans les mTEC Aire+.

\section{Mécanismes moléculaires et épigénétiques de l'expression des TRA régulés par Aire}

Les $\mathrm{mTEC}$ matures possèdent la fascinante capacité d'exprimer plusieurs milliers de TRA [1]. Une étude récente basée sur des expériences de séquençage à haut débit a en effet montré que les mTEC matures expriment $85 \%$ du génome murin soit 19293 gènes [12]. À l'heure actuelle, un seul facteur de transcription a été identifié pour réguler l'expression d'un grand nombre de TRA. Ce facteur, exclusivement localisé dans le noyau des $\mathrm{mTEC}$, correspond à la protéine Aire (Figure 2A). Des mutations de ce gène sont responsables du syndrome auto-immun APECED (autoimmune polyendocrinopathy candidiasis ectodermal dystrophy). Cette maladie rare repose généralement sur l'association de deux des trois symptômes cliniques suivants: candidose cutanéomuqueuse chronique, hypoparathyroïdie et insuffisance rénale d'origine auto-immune [20]. Les souris déficientes pour le gène Aire présentent des signes cliniques proches de ceux observés chez l'homme atteint de APECED [3]. Récemment, il a été montré que Aire est capable de réguler à lui seul l'expression de 3980 gènes [12]. Pendant de nombreuses années, plusieurs groupes de recherche ont essayé de comprendre comment un seul facteur de transcription parvient à contrôler l'expression d'autant de gènes. Plusieurs travaux ont maintenant permis d'appréhender son mode d'action.

Le gène Aire code pour une protéine de 58 kDa qui possède de multiples domaines caractéristiques de protéines capables de lier la chromatine et de réguler la transcription (Figure 2B) [21]. À l'extrémité aminoterminale, Aire possède une région HSR (homogeneously staining region) qui contient un domaine CARD (caspase recruitment domain) permettant son oligomérisation. 
Chez les patients atteints d'APECED, des mutations dans le domaine HSR de Aire perturbent la formation d'oligomères, essentielle pour sa fonction [22]. Le domaine HSR est suivi d'un domaine NLS (nuclear localization signal) responsable de la localisation nucléaire de Aire, qui est aussi assurée par les importines $\alpha 1, \alpha 3$ et $\alpha 5$ [23]. Aire contient également un domaine SAND dont la région protéique allant des acides aminés 189 à 197 possède la capacité de se lier à des séquences spécifiques de l'ADN [24]. Cependant, cette interaction ne semble pas être impliquée dans la transcription des gènes codant pour les TRA [25]. Aire contient aussi deux domaines PHD (plant homeodomain) : PHDl et PHD2. Ces séquences conservées sont retrouvées dans des protéines nucléaires impliquées dans la régulation de la transcription par reconnaissance de marques épigénétiques. Le domaine PHDl reconnaît la lysine 4 non méthylée de l'histone H3 (H3K4me0). La reconnaissance de cette marque épigénétique est nécessaire à la transcription des TRA [25-27] (Figure 2B). Le domaine PHD2, quant à lui, n'interagit pas directement avec le nucléosome mais contribue à la structure protéique tridimensionnelle de Aire qui est essentielle à son activité transcriptionnelle [28]. Le domaine PHDl est aussi impliqué dans l'interaction de Aire avec la kinase nucléaire, DNA-PK (DNA dependent protein kinase). Cette enzyme reconnaît un variant d'histone $\mathrm{H} 2 \mathrm{AX}$ phosphorylé sur la sérine 139 ( $\gamma \mathrm{H} 2 \mathrm{AX}$ ), qui se trouve être enrichi au niveau des cassures double brin de I'ADN [25] (Figure 2B). Aire contient également un domaine riche en proline (PRR) caractéristique d'autres protéines impliquées dans le contrôle de la transcription. Enfin, à son extrémité carboxy-terminale, Aire possède un domaine TAD (transcriptionnal activation domain) qui est nécessaire à l'interaction avec le facteur d'élongation P-TEFb (positive transcription elongation factor) [29].

Les connaissances acquises sur la structure de Aire ainsi que l'identification de ses différents partenaires ont permis de proposer un modèle de régulation de l'expression des TRA. À l'état basal, la machinerie transcriptionnelle est déjà en place sur les promoteurs des gènes cibles de Aire. L'ARN polymérase II (ARNpol II) est en état de pause et génère des transcrits instables de petites tailles dits «abortifs » [30]. Dans les mTદC matures, Aire reconnaît, par l'intermédiaire de son domaine PHDl, l'histone hypométhylée H3K4 (H3K4me0) dans les régions promotrices des TRA $[26,27]$ (Figure 2C). Une étude in vivo a récemment montré que le site d'initiation de la transcription des gènes codant pour les TRA régulés par Aire est aussi enrichi d'une marque répressive de la transcription : la triméthylation de l'histone H3K27 (H3K27me3) [12]. Ces données suggèrent que cette marque épigénétique pourrait être également associée au recrutement de Aire au niveau des promoteurs de ses gènes cibles. La DNA-PK, quant à elle recrutée sur les histones $\gamma \mathrm{H} 2 \mathrm{AX}$, phosphoryle Aire en interagissant avec son domaine PHDl [31]. Cette phosphorylation permet le recrutement du facteur P-TEFb via le domaine TAD de Aire. Ce facteur d'élongation phosphoryle à son tour le domaine carboxy-terminal (CTD) de I'ARNpol II, ce qui entraîne l'élongation de la transcription des gènes codant pour les TRA (Figure 2C). Aire interagit également avec le coactivateur transcriptionnel CBP (CREB-binding protein) qui acétyle les histones H3, favorisant ainsi l'accessibilité de la chromatine à la machinerie transcriptionnelle [32]. De manière intéressante, un nouveau partenaire de Aire, la protéine kinase HIPK2 (homeodomain interacting protein kinase 2) a été récemment identifiée [33]. Cette kinase régule la fonction de Aire en réprimant par phosphorylation son rôle de coactivateur de la transcription (Figure 2C). Enfin, une nouvelle étude a identifié 51 partenaires potentiels de Aire qui sont préférentiellement impliqués dans l'élongation de la transcription [34].

Aire est donc capable de réguler l'expression de milliers de TRA dans les mTEC, non pas en reconnaissant des motifs spécifiques au niveau des promoteurs de ses gènes cibles, mais en libérant l'ARNpol II de son état de pause, via une cascade de phosphorylation entre ses différents partenaires [30].

\section{Les différents rôles de Aire dans l'induction de la tolérance centrale des cellules $T$}

Aire a été initialement identifié en tant que facteur de transcription régulant l'expression de nombreux TRA dans les mTEC [3]. L'utilisation de modèles murins transgéniques a montré un rôle de Aire dans l'élimination des thymocytes autoréactifs $\mathrm{CD}^{+}{ }^{+}$et CD8 ${ }^{+}$, processus appelé « sélection négative » [35]. Ces observations sont basées sur l'utilisation de souris transgéniques OT-II. Celles-ci possèdent des thymocytes $\mathrm{CD}^{+}$qui expriment un TCR ( $T$ cell receptor) reconnaissant un peptide dérivé de l'ovalbumine (OVA) qui n'est pas normalement exprimé par les mTEC. Les thymocytes OT-II sont éliminés lorsque ces souris sont croisées avec des animaux transgéniques Rip-mOVA, qui expriment I'OVA dans les mTEC de façon spécifique. En revanche, lorsque les souris Rip-mOVA $x$ OT-II sont sur fonds génétique Aire ${ }^{-/-}$, la sélection négative des thymocytes OT-II est défectueuse. Aire joue donc un rôle clé dans la sélection négative des thymocytes $\mathrm{CD}^{+}$(Figure 3 ). Une étude utilisant des souris transgéniques, chez lesquelles l'expression des molécules de $\mathrm{CMH}$ II est réduite spécifiquement dans les mTEC Aire ${ }^{+}$, a montré que ces cellules sont de véritables cellules présentatrices d'antigènes, et qu'elles jouent donc un rôle autonome dans l'élimination des cellules $\mathrm{CD}^{+}[36]$. Le rôle de Aire a été également étudié dans la sélection négative des thymocytes $C D 8^{+}$ à l'aide de souris transgéniques OT-I qui possèdent des thymocytes $C D 8^{+}$reconnaissant I'OVA dans le contexte des molécules de CMH I [35]. Les souris RipmOVA x OT-I sur fonds Aire - $^{-/}$présentent un défaut de sélection négative des thymocytes $0 \mathrm{~T}-\mathrm{I}$, indiquant que Aire est aussi impliqué dans la sélection négative des thymocytes $\mathrm{CD}^{+}$(Figure 3 ). 


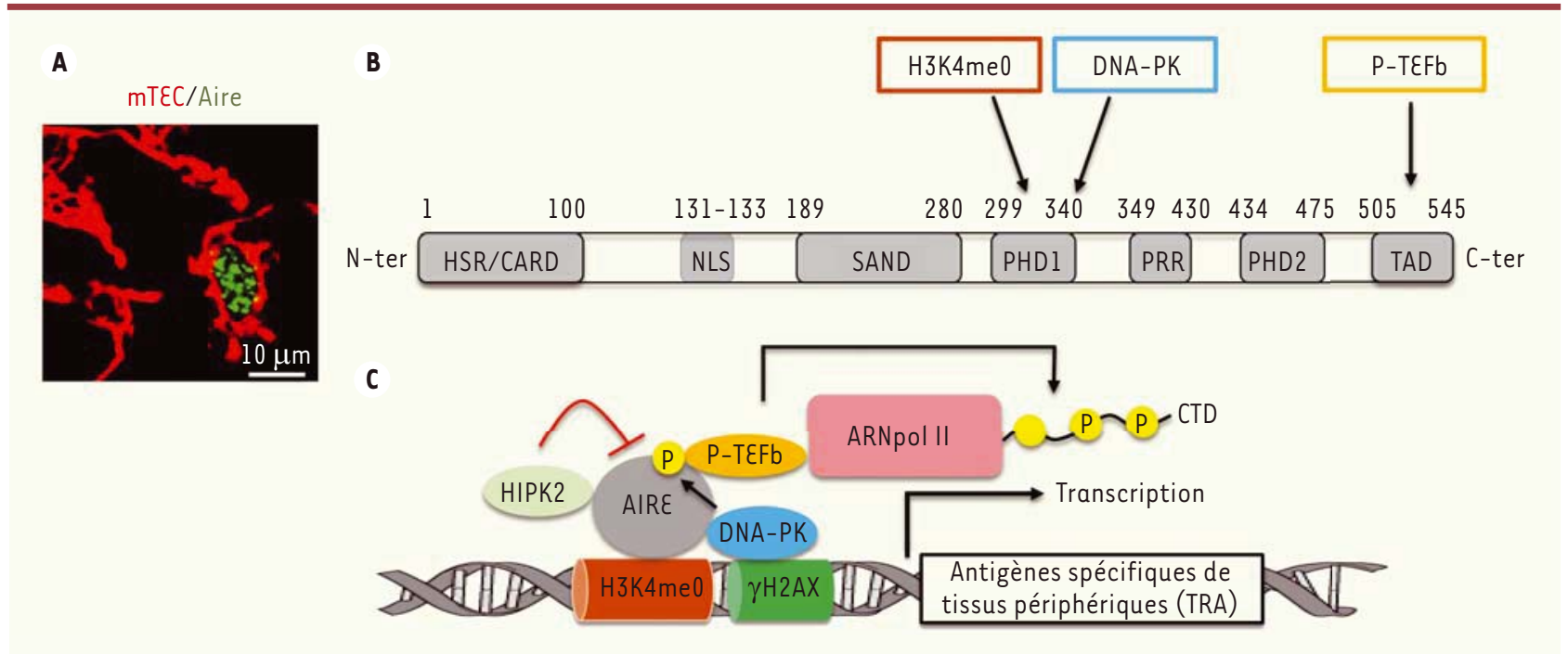

Figure 2. Mécanismes moléculaires et épigénétiques de l'expression des TRA régulés par le facteur de transcription Aire. A. Section de thymus d'une souris sauvage marquée par immunofluorescence avec un anticorps anti-kératine 14 (rouge) qui détecte les mTEC et un anticorps anti-Aire (vert). Échelle : $10 \mu \mathrm{m}$. B. Représentation schématique des différents domaines de la protéine Aire. À partir de l'extrémité amino-terminale : un domaine HSR/CARD permettant l'oligomérisation de Aire, une séquence NLS permettant sa localisation nucléaire, un domaine SAND dont le rôle reste à être caractérisé et deux domaines PHD (PHDl et PHD2). PHDl reconnaît les histones H3K4 non méthylées (H3K4me0 ; cadre rouge) et la protéine kinase dépendante de l'ADN (DNA-PK; cadre bleu). Ce domaine est suivi d'un domaine riche en proline (PRR) et d'un domaine d'activation de la transcription (TAD) qui est impliqué dans la liaison avec le facteur d'élongation P-TEFb (cadre orange). La position des acides aminés est indiquée. C. Mode d'action de Aire dans la transcription des TRA. Par son domaine PHD1, Aire est préférentiellement recruté aux promoteurs de gènes présentant des histones H3K4 non méthylées ( $\mathrm{H} 3 \mathrm{~K} 4 \mathrm{me} 0$ ). Ce domaine interagit également avec la DNA-PK qui reconnaît un variant d'histone H2AX $(\gamma \mathrm{H} 2 \mathrm{AX})$. Cette kinase phosphoryle Aire, permettant ainsi le recrutement du facteur d'élongation P-TEFb qui, à son tour, phosphoryle le domaine carboxy-terminal (CTD) de l'ARN polymérase II (ARNpol II). Cette cascade de phosphorylations entraîne la mise en route de la phase d'élongation de la transcription des TRA.

Depuis la découverte du rôle de Aire dans l'expression des TRA et dans l'élimination des thymocytes autoréactifs, plusieurs études lui ont dernièrement attribué d'autres rôles importants pour l'établissement de la tolérance centrale, notamment dans l'induction des cellules nTreg (Figure 3) [37]. Bien qu'initialement aucun défaut n'ait été observé dans la population de nTreg chez les souris Aire ${ }^{-/}$[35], une étude récente montre une diminution de ce type cellulaire dans la médulla de ces souris [38]. De plus, à l'aide d'un modèle d'adénocarcinome spontané de prostate, une autre étude indique que Aire est responsable de la génération intrathymique de cellules nTreg spécifiques d'antigènes associés aux tumeurs de la prostate [39]. II reste cependant à déterminer si l'effet de Aire observé dans cette étude s'étend à d'autres modèles tumoraux. De manière intéressante, le croisement de souris exprimant l'hémagglutinine du virus influenza (HA) sous le contrôle du promoteur de Aire (Aire-HA) avec des souris possédant un TCR transgénique qui reconnaît un peptide dérivé de HA (TCR-HA) entraîne la génération de nTreg [37]. Par conséquent, Aire joue un double rôle dans l'induction de la tolérance centrale à travers l'élimination de cellules $T$ autoréactives et la génération de cellules nTreg (Figure 3).

L'induction de la tolérance centrale des cellules T dépend de la migration des thymocytes dans la médulla au sein de laquelle ils interagissent étroitement avec les mTEC et les cellules dendritiques $[9,40]$. Aire régule l'expression de CCL (CC-type chemokine) $17 / C C L 22$ et CCL19/CCL21, ligands respectifs des récepteurs des chimiokines CCR ( $C-C$ chemokine receptor type) 4 et CCR7, qui sont nécessaires à la migration des thymocytes dans la médulla (Figure 3) [41, 42]. La déficience en CCR7 entraîne un défaut de migration des cellules $T$ au sein de la médulla, et donc un défaut dans l'induction de la tolérance centrale [43]. Par conséquent, les souris $\mathrm{CCR}^{-/-}$présentent des autoanticorps et des infiltrats lymphocytaires au sein de tissus périphériques. Elles développent ainsi des symptômes auto-immuns chroniques similaires à ceux observés chez les souris Aire ${ }^{-/}$. Aire régule aussi l'expression de la chimiokine (C class chemokine) XCLI responsable de la localisation médullaire des cellules dendritiques et ces dernières expriment le récepteur spécifique XCRI de XCLl [38]. Les souris $\mathrm{XCLI}^{-/-}$se caractérisent en effet par un défaut d'accumulation des cellules dendritiques dans la médulla. II est donc maintenant clairement établi que Aire régule l'expression de chimiokines requises pour la migration des thymocytes et des cellules dendritiques 


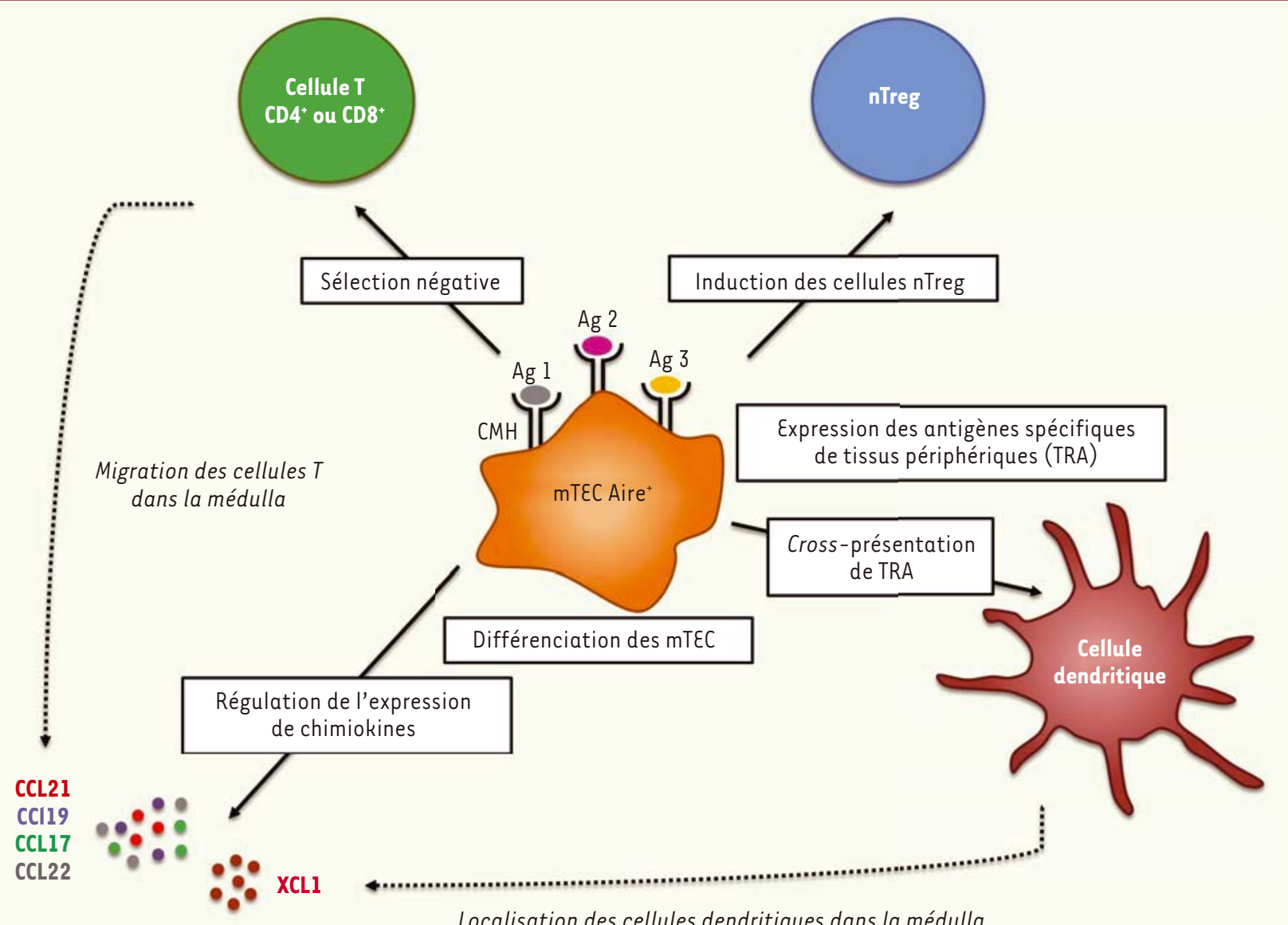

Localisation des cellules dendritiques dans la médulla

Figure 3. Les différentes fonctions de Aire dans l'induction de la tolérance des cellules T. Aire régule l'expression des gènes codant pour les TRA, il est donc impliqué dans la sélection négative des cellules T autoréactives et dans l'induction de cellules nTreg. II est aussi impliqué dans la crossprésentation des TRA par les cellules dendritiques et la différenciation des mTEC. Enfin, Aire régule l'expression de chimiokines requises pour la migration des cellules $T$ et des cellules dendritiques au sein de la médulla. Ag : antigène, CMH : complexe majeur d’histocompatibilité.

dans la médulla, processus essentiel à l'éducation des cellules T. De plus, Aire semble être impliqué dans la cross-présentation ${ }^{2}$ des TRA par les cellules dendritiques, mécanisme qui pourrait améliorer l'efficacité de la sélection négative (Figure 3) [44]. Enfin, comme discuté précédemment, Aire module aussi la différenciation terminale des mTદC.

Aire a été initialement décrit comme un facteur de transcription ayant l'étonnante capacité de réguler l'expression de milliers de TRA, nécessaires à l'élimination de thymocytes autoréactifs. De plus, Aire renforce la tolérance centrale en étant impliqué à la fois dans la génération de cellules nTreg, l'expression de chimiokines nécessaires à la migration des thymocytes et des cellules dendritiques au sein de la médulla, ainsi que dans la cross-présentation des TRA par les cellules dendritiques. Ces différentes fonctions confèrent donc au facteur de transcription Aire un rôle unique dans l'établissement de la tolérance centrale. Audelà de ce rôle, Aire semble être également impliqué dans le maintien

${ }^{2}$ Cross-présentation (présentation croisée) : capacité des cellules présentatrices de l'antigène à capturer et à présenter des antigènes exprimés par un autre type cellulaire. de la tolérance en périphérie. Aire est en effet exprimé par un sous-type de cellules stromales dans les organes lymphoïdes secondaires. Il semblerait de plus avoir la capacité d'inactiver l'activité de lymphocytes CD4 ${ }^{+}$ autoréactifs qui ont échappé au processus de sélection thymique [45]. $\diamond$

\section{SUMMARY}

Induction of central tolerance by the factor Aire: molecular and epigenetic regulation

The establishment of thymic central tolerance is a critical process to prevent the development of autoimmune diseases. Medullary thymic epithelial cells (mTEC) are essential to this process through the expression of the transcription factor Aire, which controls the transcription of many genes encoding tissue-restricted antigens. Mutations in the Aire gene are responsible for a rare autoimmune disorder called APECED (autoimmune 
polyendocrinopathy candidiasis ectodermal dystrophy). This review summarizes our current knowledge on the mode of action of Aire at the molecular and epigenetic levels in controlling the expression of tissuerestricted antigens. We also discuss recently described additional roles of this transcription factor in the induction of central T-cell tolerance. $\diamond$

\section{REMERCIEMENTS}

Ce travail a été soutenu par le programme Marie Curie Career integration grant (Marie Curie CIG_SIGnEPI4ToL_618541) attribué à Magali Irla. Les travaux de recherche dans l'équipe de Pierre Ferrier au CIML sont financés par des dotations institutionnelles de l'Inserm et du CNRS, ainsi que par des contrats de recherche spécifiques de l'Agence nationale de la recherche (ANR), de l'Institut national du cancer (INCa), de l'ITMO cancer-Alliance nationale pour les sciences de la vie et de la santé (AVIESAN), et de la Fondation Princesse Grace de la Principauté de Monaco. Noëlla Lopes est financée par une bourse doctorale du président de l'université Aix-Marseille.

\section{LIENS D'INTÉRÊT}

Les auteurs déclarent n'avoir aucun lien d'intérêt concernant les données publiées dans cet article.

\section{RÉFÉRENCES}

1. Derbinski J, Schulte A, Kyewski B, Klein L. Promiscuous gene expression in medullary thymic epithelial cells mirrors the peripheral self. Nat Immunol $2001 ; 2$ : 1032-9.

2. Sakaguchi S, Yamaguchi T, Nomura T, Ono M. Regulatory T cells and immune tolerance. Cell 2008; $133: 775-87$.

3. Anderson MS, Venanzi $\varepsilon S$, Klein L, et al. Projection of an immunological self shadow within the thymus by the aire protein. Science $2002 ; 298: 1395-401$.

4. Hamazaki Y, Fujita H, Kobayashi T, et al. Medullary thymic epithelial cells expressing Aire represent a unique lineage derived from cells expressing claudin. Nat Immunol $2007 ; 8$ : 304-11.

5. Sekai M, Hamazaki $Y$, Minato N. Medullary thymic epithelial stem cells maintain a functional thymus to ensure lifelong central T cell tolerance. Immunity $2014 ; 41: 753-61$.

6. Irla M, Hollander $G$, Reith $W$. Control of central self-tolerance induction by autoreactive CD $4^{+}$ thymocytes. Trends Immunol $2010 ; 31: 71-9$.

7. Gray D, Abramson J, Benoist C, Mathis D. Proliferative arrest and rapid turnover of thymic epithelial cells expressing Aire. J Exp Med 2007 ; 204 : 2521-8.

8. Rossi SW, Kim My, Leibbrandt A, et al. RANK signals from $\mathrm{CD4}^{+} 3^{-}$inducer cells regulate development of Aire-expressing epithelial cells in the thymic medulla.J Exp Med 2007 ; 204 : 1267-72.

9. Irla M. Cellules épithéliales médullaires thymiques exprimant Aire : acteurs clés dans l'induction de la tolérance des cellules T. Med Sci (Paris) 2012 ; 28 : 146-9.

10. Irla M, Hugues S, Gill J, et al. Autoantigen-specific interactions with $C D 4^{+}$thymocytes control mature medullary thymic epithelial cell cellularity. Immunity $2008 ; 29: 451-63$.

11. Irla M, Guerri L, Guenot J, et al. Antigen recognition by autoreactive $\mathrm{CD}^{+}$thymocytes drives homeostasis of the thymic medulla. PLoS One $2012 ; 7$ : e52591.

12. Sansom SN, Shikama-Dorn N, Zhanybekova S, et al. Population and single-cell genomics reveal the Aire dependency, relief from Polycomb silencing, and distribution of self-antigen expression in thymic epithelia. Genome Res $2014 ; 24: 1918-31$.

13. Nishikawa Y, Hirota F, Yano M, et al. Biphasic Aire expression in early embryos and in medullary thymic epithelial cells before end-stage terminal differentiation. J Exp Med 2010 ; 207 : 963-71.

14. Metzger TC, Khan IS, Gardner JM, et al. Lineage tracing and cell ablation identify a post-Aireexpressing thymic epithelial cell population. Cell Rep $2013 ; 5: 166-79$.

15. Wang $X$, Laan $M$, Bichele $R$, et al. Post-Aire maturation of thymic medullary epithelial cells involves selective expression of keratinocyte-specific autoantigens. Front Immunol $2012 ; 3: 19$.

16. Yano M, Kuroda N, Han H, et al. Aire controls the differentiation program of thymic epithelial cells in the medulla for the establishment of self-tolerance. J Exp Med 2008 ; $205: 2827-38$.

17. Watanabe $\mathrm{N}$, Wang $\mathrm{YH}$, Lee $\mathrm{HK}$, et al. Hassall's corpuscles instruct dendritic cells to induce CD4+CD25+ regulatory T cells in human thymus. Nature $2005 ; 436: 1181-5$.

18. Gillard GO, Dooley J, Erickson M, et al. Aire-dependent alterations in medullary thymic epithelium indicate a role for Aire in thymic epithelial differentiation. J Immunol 2007 ; 178 : 3007-15.

19. Dooley J, Erickson M, Farr AG. Alterations of the medullary epithelial compartment in the Airedeficient thymus: implications for programs of thymic epithelial differentiation. J Immunol 2008 ; $181: 5225-32$.

20. Mathis D, Benoist C. A decade of AIRE. Nat Rev Immunol 2007 ; 7 : 645-50.

21. Aaltonen J, Bjorses P. Cloning of the APECED gene provides new insight into human autoimmunity. Ann Med $1999 ; 31$ : 111-6.
22. Halonen $M$, Kangas $H$, Ruppell $T$, et al. APECED-causing mutations in AIRE reveal the functional domains of the protein. Hum Mutat $2004 ; 23: 245-57$.

23. Ilmarinen T, Melen $\mathrm{K}$, Kangas $\mathrm{H}$, et al. The monopartite nuclear localization signal of autoimmune regulator mediates its nuclear import and interaction with multiple importin alpha molecules. FEBS J $2006 ; 273: 315-24$.

24. Purohit S, Kumar PG, Laloraya M, She JX. Mapping DNA-binding domains of the autoimmune regulator protein. Biochem Biophys Res Commun 2005; 327 : 939-44.

25. Zumer K, Low AK, Jiang H, et al. Unmodified histone H3K4 and DNAdependent protein kinase recruit autoimmune regulator to target genes. Mol Cell Biol $2012 ; 32$ : 1354-62.

26. Koh AS, Kuo AJ, Park Sy, et al. Aire employs a histone-binding module to mediate immunological tolerance, linking chromatin regulation with organspecific autoimmunity. Proc Natl Acad Sci USA 2008 ; 105 : 15878-83.

27. Org T, Chignola F, Hetényi $C$, et al. The autoimmune regulator PHD finger binds to non-methylated histone H3K4 to activate gene expression. EMBO Rep 2008; $9: 370-6$.

28. Gaetani M, Matafora V, Saare M, et al. AIRE-PHD fingers are structural hubs to maintain the integrity of chromatin-associated interactome. Nucleic Acids Res 2012 ; 40 : 11756-68.

29. Zumer K, Plemenitas A, Saksela K, Peterlin BM. Patient mutation in AIRE disrupts $\mathrm{P}$-TEFb binding and target gene transcription. Nucleic Acids Res $2011 ; 39$ : 7908-19.

30. Giraud M, Yoshida H, Abramson J, et al. Aire unleashes stalled RNA polymerase to induce ectopic gene expression in thymic epithelial cells. Proc Natl Acad Sci USA 2012 ; 109 : 535-40.

31. Liiv I, Rebane A, Org T, et al. DNA-PK contributes to the phosphorylation of AIRE: importance in transcriptional activity. Biochim Biophys Acta 2008 ; $1783: 74-83$.

32. Peterson P, Org T, Rebane A. Transcriptional regulation by AIRE: molecular mechanisms of central tolerance. Nat Rev Immunol 2008 ; 8 : 948-57.

33. Rattay K, Claude J, Rezavandy $\varepsilon$, et al. Homeodomain-interacting protein kinase 2, a novel autoimmune regulator interaction partner, modulates promiscuous gene expression in medullary thymic epithelial cells. J Immunol $2015 ; 194: 921-8$.

34. Giraud M, Jmari N, Du L, et al. An RNAi screen for Aire cofactors reveals a role for $\mathrm{Hnrnpl}$ in polymerase release and Aire-activated ectopic transcription. Proc Natl Acad Sci USA 2014 ; 111 : 1491-6.

35. Anderson MS, Venanzi ES, Chen Z, et al. The cellular mechanism of Aire control of T cell tolerance. Immunity $2005 ; 23: 227-39$.

36. Hinterberger $M$, Aichinger M, Prazeres da Costa 0 , et al. Autonomous role of medullary thymic epithelial cells in central $\mathrm{CD}^{+} \mathrm{T}$ cell tolerance. Nat Immunol $2010 ; 11: 512-9$.

37. Aschenbrenner K, D'Cruz LM, Vollmann $\varepsilon H$, et al. Selection of Foxp $3^{+}$ regulatory $T$ cells specific for self antigen expressed and presented by Aire ${ }^{+}$ medullary thymic epithelial cells. Nat Immunol 2007 ; 8 : 351-8.

38. Lei Y, Ripen AM, Ishimaru N, et al. Aire-dependent production of XCLI mediates medullary accumulation of thymic dendritic cells and contributes to regulatory T cell development. J Exp Med 2011 ; 208 : 383-94.

39. Malchow S, Leventhal DS, Nishi S, et al. Aire-dependent thymic development of tumor-associated regulatory T cells. Science 2013 ; 339 : 1219-24.

40. Takahama Y. Journey through the thymus: stromal guides for T-cell development and selection. Nat Rev Immunol $2006 ; 6$ : 127-35.

41. Laan M, Kisand K, Kont V, et al. Autoimmune regulator deficiency results in decreased expression of CCR4 and CCR7 ligands and in delayed migration of $\mathrm{CD}^{+}$thymocytes. J Immunol 2009; 183 : 7682-91.

42. Ueno T, Hara K, Willis MS, et al. Role for CCR7 ligands in the emigration of newly generated T lymphocytes from the neonatal thymus. Immunity 2002 ; $16: 205-18$.

43. Kurobe H, Liu C, Ueno T, et al. CCR7-dependent cortex-to-medulla migration of positively selected thymocytes is essential for establishing central tolerance. Immunity $2006 ; 24: 165-77$.

44. Hubert FX, Kinkel SA, Davey GM, et al. Aire regulates the transfer of antigen from $m T \varepsilon C s$ to dendritic cells for induction of thymic tolerance. Blood 2011 ; $118: 2462-72$.

45. Gardner JM, Metzger TC, McMahon EJ, et al. Extrathymic Aire-expressing cells are a distinct bone marrow-derived population that induce functional inactivation of CD4 $\mathrm{T}$ cells. Immunity $2013 ; 39: 560-72$.

\section{TIRÉS À PART}

M. Irla 